\title{
Ultra-compact silicon photonic integrated interferometer for swept-source optical coherence tomography
}

\author{
Günay Yurtsever, ${ }^{1,2, *}$ Nicolás Weiss, ${ }^{3}$ Jeroen Kalkman, ${ }^{4}$ Ton G. van Leeuwen, ${ }^{3}$ Roel Baets,,${ }^{1,2}$ \\ ${ }^{I}$ Photonics Research Group, Ghent University-imec, Sint-Pietersnieuwstraat 41, B-9000 Ghent, Belgium \\ ${ }^{2}$ Center for Nano- and Biophotonics (NB-Photonics), Ghent University, Ghent, Belgium \\ ${ }^{3}$ Biomedical Engineering \& Physics, Academic Medical Center, University of Amsterdam, P.O. Box 22700, \\ Amsterdam 1100 DE, The Netherlands \\ ${ }^{4}$ Department of Imaging Physics, Delft University of Technology, Lorentzweg 1, 2628 CJ Delft, The Netherlands \\ *Corresponding author:gunay@intec.ugent.be
}

Received Month X, XXXX; revised Month X, XXXX; accepted Month X, XXXX; posted Month X, XXXX (Doc. ID XXXXX); published Month X, XXXX

\begin{abstract}
We demonstrate an ultra-compact silicon integrated photonic interferometer for swept-source optical coherence tomography (SS-OCT). The footprint of the integrated interferometer is only $0.75 \times 5 \mathrm{~mm}^{2}$. The design consists of three $2 \times 2$ splitters, a $13 \mathrm{~cm}$ physical length (50.4 cm optical length) reference arm and grating couplers. The photonic integrated circuit was used as the interferometer of a SS-OCT system. The sensitivity of the system was measured to be $-62 \mathrm{~dB}$ with $115 \mu \mathrm{W}$ power delivered to the sample. Using the system, we demonstrate cross-sectional OCT imaging of a layered tissue phantom. We also discuss potential improvements in passive silicon photonic integrated circuit design and integration with active components. (C) 2014 Optical Society of America

OCIS Codes: (110.4500) Optical coherence tomography, (170.4500) Optical coherence tomography, (100.3175) Interferometric imaging, (130.0130) Integrated optics.

http://dx.doi.org/10.1364/OL.99.099999
\end{abstract}

Optical coherence tomography (OCT) has become a standard tool for retinal imaging in a relatively short time since its invention [1]. During this period, the development of OCT has significantly benefited from the availability of optical fiber components developed for telecom applications. Currently, photonic integration in telecommunications is driven by the increasing demand for higher data bandwidth at a lower cost. Similarly, integrated photonics has the potential to reduce the size and the cost of OCT systems, which can open new opportunities for its deployment in various application areas.

Recently, various approaches to use integrated photonics for OCT have been studied. Akca et al. demonstrated an integrated spectrometer and a 2x2 splitter using silicon oxynitride ( $\mathrm{SiON}$ ) waveguides [2], albeit without on-chip integration of the reference arm. Nguyen et al. reported a Michelson interferometer using silicon nitride $\left(\mathrm{Si}_{3} \mathrm{~N}_{4}\right)$ strip waveguides. In this study, the reference arm was realized on chip [3]. However, the relatively short length of the reference arm did not encompass space for a sample arm galvo-scanner, and therefore the sample needed to be scanned to obtain crosssectional images. More recently, Yurtsever et al. reported a Mach-Zehnder interferometer using silicon nitride $\left(\mathrm{Si}_{3} \mathrm{~N}_{4}\right)$ box-shaped waveguides with a long on-chip reference arm capable of accommodating a galvo-scanner in the sample arm [4].

In recent years, photonic integrated circuits based on silicon have gained substantial interest from the telecom industry. Silicon is one of the most developed material systems for microelectronic fabrication. Hence, using silicon as a platform for photonic integrated circuits enables the use of existing CMOS infrastructure and processing recipes developed for silicon microelectronics fabrication. Wafer scale fabrication of silicon photonic integrated circuits using CMOS infrastructure [5] significantly reduces the price per chip. In addition to cost advantages, silicon waveguides have a very high confinement factor, which enables much smaller integrated photonic circuits than low-index-contrast material systems. In addition to miniaturization, significant reduction in size can enable faster, parallel OCT systems. Furthermore, several groups have also demonstrated that active components such as lasers, photodetectors, and modulators can be integrated on wafer-scale with silicon waveguides. A broad review of active component integration with silicon waveguides is presented by Park et al. [6]. In addition to active components, electronic and photonic integration can be combined on the same material system, which can further miniaturize electro-optical systems [7]. Thus, compared to other integrated photonic material systems, silicon can have a significant potential for mass fabrication of OCT devices, which would result in miniaturization, cost reduction, and wider deployment.

In this study, for the first time to our knowledge, we demonstrate OCT imaging based on a silicon photonic integrated chip. The size of the chip is only $0.75 \times 5 \mathrm{~mm}^{2}$, which is 90 times smaller than the chip we previously demonstrated in silicon nitride $\left(\mathrm{Si}_{3} \mathrm{~N}_{4}\right)$ [4]. The chip consists of grating couplers, a $13 \mathrm{~cm}$ long (physical length) reference arm, and three $2 \times 2$ couplers in a Mach-Zehnder interferometer configuration. Using a swept light source, we obtained cross-sectional OCT images from a layered tissue phantom by scanning the beam with a 
galvo-scanner. Besides the design and the measurements, we discuss the challenges and potential improvements in silicon photonic integrated circuit design related to OCT imaging.

An illustration of the OCT system used to perform the experiments is shown in Fig. 1(a). Light from a swept source (Axsun Technologies, USA) centered at $1312 \mathrm{~nm}$ is coupled into the chip using an SMF-28 fiber array with a $127 \mu \mathrm{m}$ pitch and $10^{\circ}$ polish angle (OZ Optics, Canada). For long term alignment stability, the fiber array was glued to the chip with UV curable glue. Coupling in and out of the chip is achieved with tilted grating couplers with reduced back reflection [8]. Grating couplers provide access to waveguides through vertical coupling. The polarization of the input light is adjusted to the TEpolarization since the grating couplers were designed for TE-light. After entering the chip, the light is split into reference and sample arms via the $2 \times 2$ coupler $\mathrm{C} 1$. In the sample arm, the light is split again by the $2 \times 2$ coupler $\mathrm{C} 2$ and finally sent out of the chip with a grating coupler. The light exiting the chip is coupled to a short piece of $(2 \mathrm{~cm})$ single mode fiber (SMF), which is attached to a collimator (F280APC-C, Thorlabs, USA). The light from the collimator is directed to the sample via the galvo-scanner and the scan lens. Back scattered light from the sample is combined on the chip with the light from the reference arm at the $2 \times 2$ coupler C3. The combined light at both output arms of the $2 \times 2$ coupler C3 leaves the chip via grating couplers. The fibers in the fiber array aligned with these grating couplers send the light to a balanced photodetector (Thorlabs PDB 110C, USA).

A microscope image of the fabricated chip is shown in Fig. 1(b). The single mode rib waveguides are $470 \mathrm{~nm}$ wide, $220 \mathrm{~nm}$ high, and the bend radius is $50 \mu \mathrm{m}$. The loss of the straight waveguides is measured to be $0.35 \mathrm{~dB} / \mathrm{cm}$. The group index of the waveguides is $\mathrm{n}_{\text {group }}=3.88$ and hence the optical length of the reference arm is $50.4 \mathrm{~cm}$. The designed circuit was fabricated on a $200 \mathrm{~mm}$ silicon-on-insulator (SOI) wafer consisting of $220 \mathrm{~nm}$ thick silicon on top of $2000 \mathrm{~nm}$ of buried oxide layer using $248 \mathrm{~nm}$ lithography. A single etch step of $70 \mathrm{~nm}$ was used for the definition of the waveguides and the grating couplers. To protect the chip from dust it was covered with silicon dioxide as a top cladding by using plasma deposition. The fabrication was performed through a cost sharing multi-project wafer service, ePIXfab silicon photonics platform [9].

To characterize the individual photonic integrated components, we measured the bandwidth of the grating couplers, the $2 \times 2$ couplers, and the power levels at the sample and reference arms. The grating couplers have $7.5 \mathrm{~dB}$ insertion loss and a $45 \mathrm{~nm} 3 \mathrm{~dB}$ bandwidth. For the $2 \times 2$ couplers, we chose to design adiabatic couplers as they have a broader bandwidth than directional couplers. Adiabatic couplers using both rib and ridge silicon waveguides have been experimentally demonstrated [10, 11]. In principle, couplers $\mathrm{C} 1$ and $\mathrm{C} 2$ can be realized using $\mathrm{y}^{-}$splitters, as they are wavelength independent. However, generally $\mathrm{y}^{-}$-splitters have higher insertion losses than directional couplers and also cause some back reflection due to fabrication limited blunt at the junction. The length of the $2 \times 2$ couplers is $2.1 \mathrm{~mm}$ and the gap between the waveguides is $0.7 \mu \mathrm{m}$. Due to deviations between the designed and the fabricated geometry, the splitting ratio deviated from simulations; the measured splitting ratio was $45: 55$ at $1266 \mathrm{~nm}$ and 30:70 at $1356 \mathrm{~nm}$.

The swept source has a $50 \mathrm{kHz}$ repetition rate, $92 \mathrm{~nm}$ tuning range, and provides $-6 \mathrm{~dB}$ roll-off at $3.5 \mathrm{~mm}$ depth. Each sweep cycle is sampled with 1088 points which corresponds to a $5.09 \mathrm{~mm}$ maximum imaging depth. The power delivered to the chip was $12.4 \mathrm{dBm}(17.5 \mathrm{~mW})$, while the power on the sample was measured to be $-9.4 \mathrm{dBm}(115 \mu \mathrm{W})$, which means $21.8 \mathrm{~dB}$ attenuation compared to the input power. The attenuation is due to losses induced by the two grating couplers and two $2 \times 2$ couplers. The power levels from the reference arm reaching the balanced photodetector were $-14.7 \mathrm{dBm}$ $(36 \mu \mathrm{W})$ and $-16.8 \mathrm{dBm}(21 \mu \mathrm{W})$.

Owing to their lower loss, the rib waveguides in this design ( $0.35 \mathrm{~dB} / \mathrm{cm}$ loss) were preferred over ridge waveguides ( $2 \mathrm{~dB} / \mathrm{cm}$ loss). However, the dispersion of these waveguides is significant; their group velocity dispersion was simulated to be $-1700 \mathrm{ps} /(\mathrm{nm} \cdot \mathrm{km})$ at $1310 \mathrm{~nm}$. As dispersion of the reference arm cannot be compensated for in the sample arm, numerical dispersion compensation in software is necessary. For dispersion compensation, we first measured the reflection from a mirror in the sample arm. The measured interference fringe (Fig. 2(a)) shows that, the fringe frequency increases strongly with increasing wavelength. The measured bandwidth of the interference is reduced, compared to the source bandwidth, due to the bandwidth of the grating couplers; which limits the axial resolution to $24.4 \mu \mathrm{m}$ (FWHM) (obtained from the Fourier transform of the envelope of the interference signal). On the other hand, without dispersion compensation, the Fourier transform of the interference resulted in $1231 \mu \mathrm{m}$ axial resolution as shown in Fig. 2(b). To compensate for dispersion, we found the dispersion coefficients by fitting a $3^{\text {rd }}$ order polynomial to the phase obtained from the interference. After $3^{\text {rd }}$ order dispersion compensation [12], $25.5 \mu \mathrm{m}$ axial resolution was obtained as shown in Fig. 2(b). Compensation for dispersion orders higher than the $3^{\text {rd }}$ order did not further improve the axial resolution. The axial resolution of a fiber based-OCT system using the same laser was $12.5 \mu \mathrm{m}$ [13]. It should be noted that if the sample is placed close to the maximum imaging depth, aliasing will occur due to the large dispersion. The aliased sginals cannot be compensated for dispersion and axial resolution and OCT signal amplitude near the maximum imaging depth will be degraded [14].

To characterize the sensitivity of the system we placed an OD 1.2 neutral density filter in the sample arm and measured the reflection from a mirror. The sensitivity of the system was calculated by the ratio of the amplitude of the OCT signal peak to the standard deviation of the noise floor. The sensitivity of the system with $115 \mu \mathrm{W}$ power on the sample was measured to be $-62 \mathrm{~dB}$. We also measured the sensitivity of the fiber-based SS-OCT system [13] as $84 \mathrm{~dB}$, using the same swept source, reference and sample arm powers, galvo-scanner, photodetector, and analog-todigital converter card settings. Thus, the chip based system had a $22 \mathrm{~dB}$ lower sensitivity than the fiber-based system. The degradation of the sensitivity can be 
explained mainly by the insertion losses in the grating couplers and an additional $3 \mathrm{~dB}$ loss of the $2 \times 2$ coupler $\mathrm{C} 2$ (while reflected light from the sample passes through the $2 \times 2$ coupler $\mathrm{C} 2$ and goes towards the photodetectors). Additionally, the 2x2 couplers of the fiber SS-OCT system (FC1310-70-50-APC, Thorlabs, USA) were significantly more wavelength independent than the on-chip $2 \times 2$ couplers and thus are better suited for balanced detection resulting in improved common mode noise reduction.

To demonstrate OCT imaging with the chip based system, we imaged a three-layer tissue phantom as shown in Fig. 3. The scattering layers of the phantom had a scattering coefficient $\mu_{\mathrm{s}}=4 \mathrm{~mm}^{-1}$, refractive index $\mathrm{n}=1.41$, and were separated by non-scattering tape [3] The image was obtained by averaging $100 \mathrm{~B}$-scans. Near the middle of the image, a faint residual background signal as a result of internal reflections in the chip is seen. Due to laser power and phase fluctuations between each A-line, the background signal could not be completely removed by background subtraction. The residual background signal was observed only with the chip-based system, and is absent in the fiber-based system. The phantom was placed slightly away from the residual background signal to prevent them from overlapping.

Although we demonstrated OCT imaging using the chip-based system, the sensitivity and axial resolution are relatively low compared to state-of-the art OCT systems. However, the sensitivity and the axial resolution can be significantly improved using more advanced fabrication methods to fabricate fiber to chip coupling structures with lower insertion loss and larger bandwidth. Simple grating couplers used in this study are easy to fabricate but are not the optimum solution. By using more advanced fabrication processes, grating couplers with $1.6 \mathrm{~dB}$ loss and $80 \mathrm{~nm} 3 \mathrm{~dB}$ bandwidth have been demonstrated [15]. For even larger bandwidths, a solution is to use horizontal butt-coupling using spot size converters to couple the light in and out of the chip. Spot size converters gradually enlarge the mode size in the waveguide and are usually fabricated as tapered waveguide structures. Ben Bakir et al. demonstrated $3 \times 3 \mu \mathrm{m}$ spot size converters with $>200 \mathrm{~nm}$ bandwidth and $1 \mathrm{~dB}$ insertion loss [16]. Coupling efficiently to such a small spot size converter with a lensed fiber is feasible. However, based on our experience, coupling efficiently to multiple adjacent small spot size converters may not be practical due to fabrication tolerances in lensed fiber arrays. Thus, spot size converters with low-loss coupling to standard single mode fibers (SMFs) are desirable. Shiraishi et al. demonstrated a spot size converter with $2.8 \mathrm{~dB}$ coupling loss to a standard SMF [17]. SMF spot size converters would increase the coupling tolerances and reduce packaging costs, and improve OCT performance.

In general, high index contrast integrated waveguides are much more dispersive than SMFs, however by changing the width and height of the waveguides or the cladding material, dispersion could be reduced. Through simulations, we observed that using ridge waveguides rather than rib waveguides provides more flexible dispersion tailoring. For example, we simulated that a silicon-on-insulator, $\mathrm{SiO}_{2}$ cladding ridge waveguide with $220 \mathrm{~nm}$ height and $540 \mathrm{~nm}$ width would have zero group velocity dispersion at $1310 \mathrm{~nm}$. Although rib waveguides have higher losses than ridge waveguides, their loss could be reduced to $0.45 \mathrm{~dB} / \mathrm{cm}$ by using higher resolution lithography [5].

The return loss of individual components in a photonic integrated circuit for OCT is also crucial. The return loss of fiber components which are connected to each other with fibers much longer than the coherence length of the light source do not present a significant problem. However, the distances between individual components in a photonic integrated chip are in the order of the coherence length of OCT light sources. Thus, fringes due to reflections within the chip may appear as spurious background signals in the OCT signal. To avoid this, the transition between different photonic integrated components needs to be sufficiently smooth to reduce such reflection effects.

For Fourier-domain OCT implementation, currently silicon is more appropriate for SS-OCT than spectraldomain OCT. Further technological improvements are necessary to demonstrate silicon spectrometers with specifications necessary for OCT $(\geq 512$ channels, $\leq 0.2$ $\mathrm{nm}$ resolution). While the first step towards an on-chip SS-OCT is to optimize the passive structures, in the long term, integration with active components will open new application opportunities. Wafer scale integration of germanium photodetectors on silicon has recently been demonstrated [18]. An interferometer design similar to the one presented in this study can be integrated with such on-chip photodetectors and used for balanced SSOCT. Integrating the photodetectors will also eliminate the chip-to-fiber losses for the light sent to the photodetectors. Integration of a tunable laser and other active elements (e.g. optical amplifier, modulator) can be pursued by bonding pre-fabricated components on top of the passive structures or by bonding the active material epitaxial layers on top of the passive waveguides and then processing the active components [6].

Considering state-of-the-art silicon photonic components, including integrated photodetectors, an integrated interferometer for SS-OCT with sensitivity close to fiber interferometers could be realized. The major loss (for light returning from the sample) will be the $3 \mathrm{~dB}$ loss at coupler $\mathrm{C} 2$, as broadband integrated circulators have not matured yet. Additional 1-2 $\mathrm{dB}$ loss at the chip to collimating lens interface and $0.5 \mathrm{~dB}$ propagation loss could be present.

In conclusion, we have fabricated and characterized a silicon-based, integrated photonics interferometer for swept-source OCT. We have demonstrated cross-sectional imaging of a layered tissue phantom showing the feasibility and potential of integrated silicon-based OCT systems. Thanks to the small size of silicon photonic integrated components, silicon photonics can become a platform for highly parallel OCT systems. Monolithic/hybrid integration of active components with passive structures can enable an on-chip OCT system at low cost and small form factor.

Acknowledgments

This work was supported by Gent UniversityMethusalem project "Smart Photonic Chips" and the IOP 
Photonics Devices program managed by Agentschap NL. We would like to thank Dr. Dirk J. Faber for helpful discussions.

\section{References}

1. D. Huang, E. A. Swanson, C. P. Lin, J. S. Schuman, W. G. Stinson, W Chang, M. R. Hee, T. Flotte, K. Gregory, C. A. Puliafito, and J. G. Fujimoto, Science 254, 1178 (1991).

2. B. I. Akca, B. Považay, A. Alex, K. Wörhoff, R. M. de Ridder, W. Drexler, and M. Pollnau, Opt. Express 21, 16648 (2013).

3. V. Duc Nguyen, N. Weiss, W. Beeker, M. Hoekman, A. Leinse, R. G. Heideman, T. G. van Leeuwen, and J. Kalkman, Opt. Lett. 37, 4820 (2012).

4. G. Yurtsever, B. Považay, A. Alex, B. Zabihian, W. Drexler, and R. Baets, Biomed. Opt. Express 5, 1050 (2014).

5. S. K. Selvaraja, P. De Heyn, G. Winroth, P. Ong, G. Lepage, C. Cailler, A. Rigny, K. Bourdelle, W. Bogaerts, D. VanThourhout, J. Van Campenhout, and P. Absil, in Optical Fiber Communication Conference, OSA Technical Digest (online) (Optical Society of America, 2014), paper Th2A.33.

6. H. Park, M. N. Sysak, H. W. Chen, A. W. Fang, D. Liang, L. Liao, B. R. Koch, J. Bovington, Y. Tang, K. Wong, M. Jacob-Mitos, R. Jones, and J. E. Bowers, IEEE J. Sel. Top. Quantum Electron. 17, 671 (2011).

7. J. S. Orcutt, B. Moss, C. Sun, J. Leu, M. Georgas, J. Shainline, E. Zgraggen, H. Li, J. Sun, M. Weaver, S. Urošević, M. Popović, R. J. Ram, and V. Stojanović, Opt. Express 20, 12222 (2012).

8. D. Vermeulen, Y. De Koninck, Y. Li, E. Lambert, W. Bogaerts, R. Baets, and G. Roelkens, Opt. Express 20, 22278 (2012)

9. http://www.epixfab.eu/

10. H. Yun, W. Shi, Y. Wang, L. Chrostowski and N. A. F. Jaeger, Proc. SPIE 8915, 89150V (2013).

11. M. R. Watts, J. Sun, C. DeRose, D. C. Trotter, R. W. Young, and G. N. Nielson, Opt. Lett. 38, 733 (2013)

12. M. Wojtkowski, V. Srinivasan, T. Ko, J. Fujimoto, A. Kowalczyk, and J. Duker, Opt. Express 12, 2404 (2004).

13. N. Weiss, T. G. van Leeuwen, and J. Kalkman, Physical Review E 88, 042312 (2013).

14. T. Bajraszewski, M. Wojtkowski, M. Szkulmowski, A. Szkulmowska, R. Huber, and A. Kowalczyk, Opt. Express 16, $4163(2008)$

15. D. Vermeulen, S. Selvaraja, P. Verheyen, G. Lepage, W. Bogaerts, P. Absil, D. Van Thourhout, and G. Roelkens, Opt. Express 18, 18278 (2010).

16. B. Ben Bakir, A. V. de Gyves, R. Orobtchouk, P. Lyan, C. Porzier, A. Roman, J.-M. Fedeli, IEEE Photon. Technol. Lett. 22, 739 (2010).

17. K. Shiraishi, H. Yoda, and C. S. Tsai, Opt. Express 20 24370 (2012)

18. S. Assefa, F. Xia, W. Green, C. Schow, A. Rylyakov, and Y. Vlasov, IEEE J. Sel. Topics Quantum Electron., 161376 (2010).
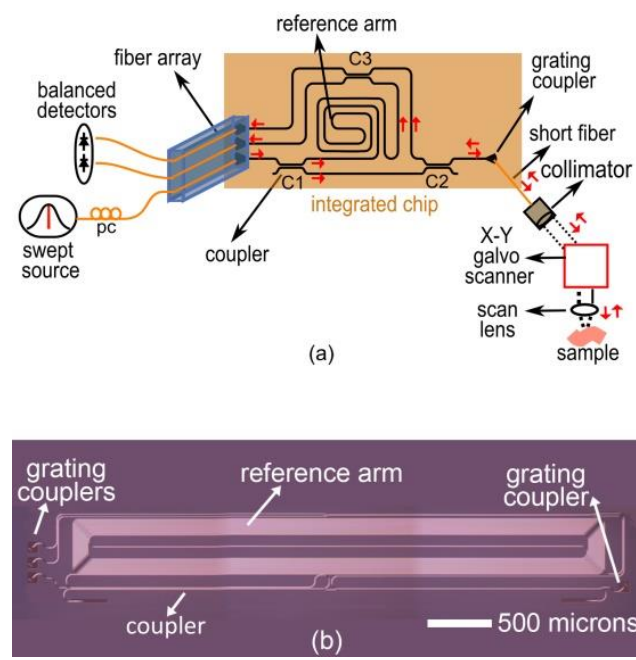

Fig. 1. (a) Schematic of the OCT setup with the photonic integrated circuit, pc: polarization controller, C1, C2, C3: $2 \times 2$ couplers. The direction of the light is indicated by red arrows (b) Microscope image of the fabricated photonic integrated interferometer.
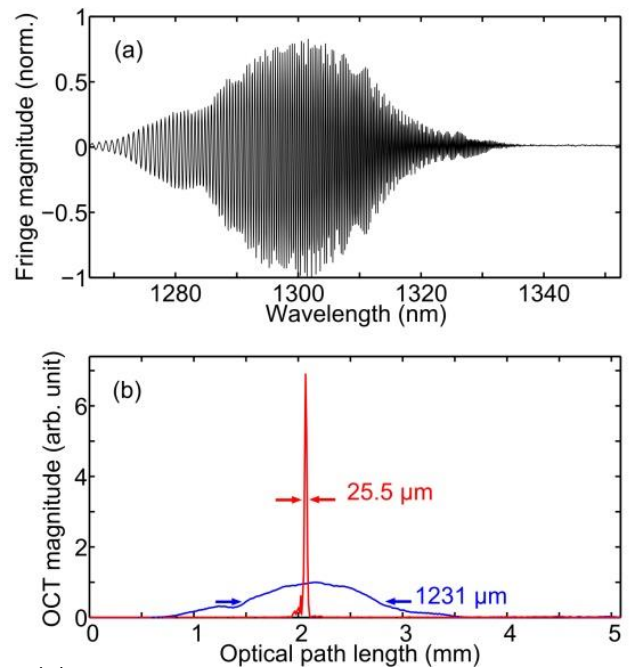

Fig. 2. (a) Interference fringe for a reflection from a mirror. (b) Fourier-transform of the interference signal; before (blue) and after (red) dispersion compensation. The peak of the blue line is normalized to 1 .

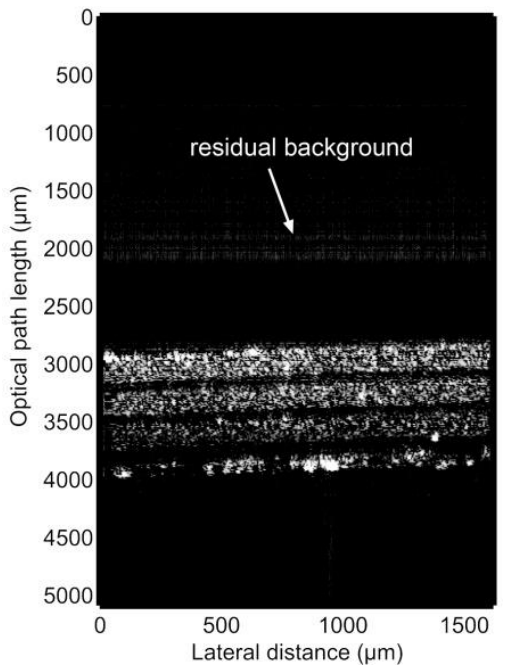

Fig. 3. OCT cross-sectional image (average of $100 \mathrm{~B}$-scans) of a layered tissue phantom. 
1. D. Huang, E. A. Swanson, C. P. Lin, J. S. Schuman, W. G. Stinson, W Chang, M. R. Hee, T. Flotte, K. Gregory, C. A. Puliafito, and J. G. Fujimoto, "Optical coherence tomography," Science 254 (5035), 1178-1181, (1991)

http://www.sciencemag.org/content/254/5035/1178.

2. B. I. Akca, B. Považay, A. Alex, K. Wörhoff, R. M. de Ridder, W. Drexler, and M. Pollnau, "Miniature spectrometer and beam splitter for an optical coherence tomography on a silicon chip," Opt. Express 21, 16648-16656 (2013)

http://www.opticsinfobase.org/oe/abstract.cfm?URI=oe-21-14-16648.

3. V. Duc Nguyen, N. Weiss, W. Beeker, M. Hoekman, A. Leinse, R. G. Heideman, T. G. van Leeuwen, and J. Kalkman,

"Integrated-optics-based swept-source optical coherence tomography," Opt. Lett. 37, 4820-4822 (2012)

http://www.opticsinfobase.org/ol/abstract.cfm?URI=ol-37-23-4820

4. G. Yurtsever, B. Považay, A. Alex, B. Zabihian, W. Drexler, and R. Baets, "Photonic integrated Mach-Zehnder

interferometer with an on-chip reference arm for optical coherence tomography," Biomed. Opt. Express 5, 1050-1061 (2014) http://www.opticsinfobase.org/boe/abstract.cfm?URI=boe-5-4-1050.

5. S. Selvaraja, P. De Heyn, G. Winroth, P. Ong, G. Lepage, C. Cailler, A. Rigny, K. Bourdelle, W. Bogaerts, D. Van

Thourhout, J. Van Campenhout, P. Absil, "Highly uniform and low-loss passive silicon photonics devices using a 300mm

CMOS platform", Optical Fiber Communication Conference (OF2014), United States, p.Th2A.33 (2014)

http://www.opticsinfobase.org/abstract.cfm?uri=OFC-2014-Th2A.33

6. H. Park, M. N. Sysak, H. W. Chen, A. W. Fang, D. Liang, L. Liao, B. R. Koch, J. Bovington, Y. Tang, K. Wong, M. JacobMitos, R. Jones, and J. E. Bowers, "Device and Integration Technology for Silicon Photonic Transmitters," IEEE J. Sel.

Top. Quantum Electron. 17(3), 671-688 (2011)

http://ieeexplore.ieee.org/xpl/login.jsp?tp=\&arnumber=5722968\&url=http $\% 3 \mathrm{~A} \% 2 \mathrm{~F} \% 2 \mathrm{Fieeexplore.ieee.org} \% 2 \mathrm{Fxpls} \% 2 \mathrm{Fabs}$ all.jsp\%3Farnumber\%3D5722968

7. J. S. Orcutt, B. Moss, C. Sun, J. Leu, M. Georgas, J. Shainline, E. Zgraggen, H. Li, J. Sun, M. Weaver, S. Urošević, M. Popović, R. J. Ram, and V. Stojanović, "Open foundry platform for high-performance electronic-photonic integration," Opt. Express 20, 12222-12232 (2012) http://www.opticsinfobase.org/oe/abstract.cfm?URI=oe-20-11-12222.

8. D. Vermeulen, Y. De Koninck, Y. Li, E. Lambert, W. Bogaerts, R. Baets, and G. Roelkens, "Reflectionless grating couplers for Silicon־on-Insulator photonic integrated circuits," Opt. Express 20, 22278-22283 (2012)

http://www.opticsinfobase.org/oe/abstract.cfm?URI=0e-20-20-22278.

9. http://www.epixfab.eu/

10. H. Yun, W. Shi, Y. Wang, L. Chrostowski and N. A. F. Jaeger, "2×2 adiabatic 3-dB coupler on silicon-on-insulator rib waveguides," Proc. SPIE 8915, 89150V (2013) http://proceedings.spiedigitallibrary.org/proceeding.aspx?articleid=1754945 11. M. R. Watts, J. Sun, C. DeRose, D. C. Trotter, R. W. Young, and G. N. Nielson, "Adiabatic thermo-optic Mach-Zehnder switch," Opt. Lett. 38, 733-735 (2013)

http://www.opticsinfobase.org/ol/abstract.cfm?URI=ol-38-5-733.

12. M. Wojtkowski, V. Srinivasan, T. Ko, J. Fujimoto, A. Kowalczyk, and J. Duker, "Ultrahigh-resolution, high-speed, Fourier domain optical coherence tomography and methods for dispersion compensation," Opt. Express 12, 2404-2422 (2004) http://www.opticsinfobase.org/oe/abstract.cfm?URI=oe-12-11-2404.

13. N. Weiss, T. G. van Leeuwen, and J. Kalkman, "Localized measurement of longitudinal and transverse flow velocities in colloidal suspensions using optical coherence tomography," Physical Review E 88, 042312 (2013)

http://journals.aps.org/pre/abstract/10.1103/PhysRevE.88.042312

14. T. Bajraszewski, M. Wojtkowski, M. Szkulmowski, A. Szkulmowska, R. Huber, and A. Kowalczyk, "Improved spectral optical coherence tomography using optical frequency comb," Opt. Express 16, 4163-4176 (2008)

http://www.opticsinfobase.org/oe/abstract.cfm?URI=oe-16-6-4163

15. D. Vermeulen, S. Selvaraja, P. Verheyen, G. Lepage, W. Bogaerts, P. Absil, D. Van Thourhout, and G. Roelkens, "Highefficiency fiber-to-chip grating couplers realized using an advanced CMOS-compatible Silicon-On-Insulator platform," Opt. Express 18, 18278-18283 (2010) http://www.opticsinfobase.org/oe/abstract.cfm?URI=oe-18-17-18278.

16. B. Ben Bakir, A.V. de Gyves, R. Orobtchouk, P. Lyan, C. Porzier, A. Roman, J.-M. Fedeli, "Low-Loss ( 1 dB) and Polarization-Insensitive Edge Fiber Couplers Fabricated on 200-mm Silicon־on-Insulator Wafers," IEEE Photon. Technol. Lett. 22(11), 739-741 (2010)

http://ieeexplore.ieee.org/xpl/login.jsp?tp=\&arnumber=5432953\&url=http $\% 3 \mathrm{~A} \% 2 \mathrm{~F} \% 2 \mathrm{Fieeexplore.iee.} \mathrm{org} \% 2 \mathrm{Fiel} 5 \% 2 \mathrm{~F} 68 \%$ 2F5458299\%2F05432953.pdf\%3Farnumber\%3D5432953

17. K. Shiraishi, H. Yoda, and C. S. Tsai, "A two-port polarization-insensitive coupler module between single-mode fiber and silicon-wire waveguide," Opt. Express 20, 24370-24375 (2012) http://www.opticsinfobase.org/oe/abstract.cfm?URI=oe$\underline{20-22-24370}$ 
18. S. Assefa, F. Xia, W. Green, C. Schow, A. Rylyakov, and Y. Vlasov, "CMOS-integrated optical receivers for on-chip interconnects," IEEE J. Sel. Topics Quantum Electron., 16(5), 1376-1385 (2010)

http://ieeexplore.ieee.org/xpl/login.jsp?tp=\&arnumber=5482019\&url=http\%3A\%2F\%2Fieeexplore.iee.org\%2Fiel5\%2F2944

\%2F4481213\%2F05482019.pdf\%3Farnumber\%3D5482019 